Arq. Bras. Med. Vet. Zootec., v.69, n.5, p.1181-1185, 2017

\title{
Abiotrofia cerebelar em um gato: relato de caso
}

\author{
[Cerebellar abiotrophy in a feline: case report] \\ M.O. Firmino ${ }^{1}$, L.Â. Maia ${ }^{1}$, C.C.F.S. Costa ${ }^{1}$, L.M. Oliveira ${ }^{2}$, A.F.M. Dantas ${ }^{1}$ \\ ${ }^{1}$ Programa de pós-graduação - Universidade Federal de Campina Grande - Patos, PB \\ ${ }^{2}$ Programa de pós-graduação - Universidade Federal Rural de Pernambuco - Recife, PE
}

\begin{abstract}
RESUMO
Um caso de abiotrofia cerebelar em um gato com 45 dias de idade foi diagnosticado no Laboratório de Patologia Animal, Hospital Veterinário da Universidade Federal de Campina Grande. O animal apresentava, havia 15 dias, apatia, anorexia, desidratação, ataxia, hipermetria, espasticidade dos membros torácicos e pélvicos, tremores de intenção, nistagmo, opistótono, déficit proprioceptivo e ausência de resposta de ameaça. Clinicamente, havia a suspeita de hipoplasia cerebelar, e, devido ao prognóstico desfavorável, o animal foi eutanasiado. Na necropsia, não foram observadas alterações macroscópicas. Microscopicamente, as lesões estavam restritas ao cerebelo e caracterizavam-se por alterações neurodegenerativas e necróticas, com desaparecimento segmentar dos neurônios de Purkinje. Nessas áreas, também se verificaram espaços em branco, denominado aspecto de cesto vazio, resultantes da perda dos neurônios de Purkinje, além de raros esferoides axonais e proliferação dos astrócitos de Bergmann. Em algumas áreas, a camada granular estava hipocelular e havia moderada gliose multifocal na camada molecular. O diagnóstico de abiotrofia cerebelar foi realizado com base nos dados epidemiológicos, clínicos e, principalmente, pelas alterações histopatológicas dos neurônios de Purkinje características da doença.
\end{abstract}

Palavras-chave: doença hereditária, disfunção cerebelar, degeneração neuronal

\begin{abstract}
The aim of this report was to describe a case of cerebellar abiotrophy in cat with 45-year-old diagnosed at the Animal Pathology Laboratory, Veterinary Hospital of the Federal University of Campina Grande. The animal had presented 15-day apathy, anorexia, dehydration and neurological signs, characterized by ataxia, hypermetria, spasticity of fore and hindlimbs, intention tremor, nystagmus, opisthotonos, proprioceptive deficits, and absence of threat response. Clinically, cerebellar hypoplasia was suspected and the animal was euthanized due to poor prognosis. During necropsy, gross lesions were not observed. Microscopically the lesions were restricted to the cerebellum and were characterized by neurodegenerative and necrotic damage with segmental disappearance of the Purkinje cells. In these areas, there were also empty spaces, called the empty basket aspect, resulting from the loss of Purkinje cells, as well as rare axonal spheroids and proliferation of Bergmann's astrocytes. In some areas, the granular layer was hypocellular and there was moderate multifocal gliosis in the molecular layer. The diagnosis of cerebellar abiotrophy was based on epidemiological, clinical and mainly on histopathological changes in neurons of Purkinje disease characteristics.
\end{abstract}

Keywords: hereditary disease, cerebellar dysfunction, neuronal degeneration

Recebido em 10 de março de 2017

Aceito em 25 de março de 2017

E-mail: millena_deoliveira@yahoo.com.br 


\section{INTRODUÇÃO}

Abiotrofia cerebelar é considerada uma doença rara, de origem congênita e hereditária. Caracteriza-se por alterações degenerativas e progressivas dos neurônios de Purkinje, que podem ser observadas após o nascimento, em várias espécies de animais e no homem (Lahunta, 1990; Riet-Correa et al., 1998; Macêdo et al., 2011). A doença foi descrita em cães (Mari et al., 2014), bovinos (Macêdo et al., 2011; Oliveira 2012), equinos (Lahunta, 1990; Foley et al., 2011), mas, na espécie felina (Shamir et al., 1999; Negrin et al., 2006; Biolatti et al., 2010), existem poucos relatos na literatura. Os sinais clínicos podem ocorrer logo após o nascimento ou mais tardiamente, caracterizados principalmente por tremores de intenção, hipermetria, espasticidade, ataxia e crises epileptiformes (Riet-Correa et al., 1998; Negrin et al., 2006; Biolatti et al., 2010).

No Brasil, a doença foi relatada em cães (Mari et al., 2014) e bovinos (Macêdo et al., 2011), não sendo identificado nenhum caso de abiotrofia cerebelar em felino doméstico na literatura. Dessa forma, objetivou-se, com este trabalho, descrever um caso de abiotrofia cerebelar em um gato, caracterizando-se os aspectos epidemiológicos, clínicos e histopatológicos.

\section{RELATO DO CASO}

A doença ocorreu em um gato, macho, sem raça definida, de 45 dias de idade, que foi atendido no Hospital Veterinário da Universidade Federal de Campina Grande, Patos-PB, com histórico de anorexia, dificuldade de manter-se em estação e andar cambaleante.

De acordo com o proprietário, duas gatas irmãs pariram quatro filhotes cada, sendo dois filhotes de cada ninhada com alterações clínicas semelhantes. Três filhotes haviam morrido, e o animal do presente relato apresentava as manifestações clínicas havia aproximadamente 15 dias.

Ao exame físico, verificou-se apatia, anorexia, desidratação e sinais neurológicos acentuados, caracterizados por ataxia, hipermetria, espasticidade dos membros torácicos e pélvicos, tremores de intenção generalizados (mais acentuados na cabeça), nistagmo, opistótono, déficit proprioceptivo e ausência de resposta de ameaça (Fig. 1A). No "head raising test" (Fig. 1B), o animal perdeu totalmente o equilíbrio, caindo logo em seguida e demonstrando espasticidade dos membros e cauda (posição atípica) (Fig. 1C), o que indica um quadro de disfunção cerebelar.

Devido à suspeita clínica de disfunção cerebelar e ao prognóstico desfavorável, optou-se pela eutanásia do animal. Na necropsia, não foram observadas alterações macroscópicas no sistema nervoso central (Fig. 1D) e nos demais órgãos. Fragmentos de órgãos das cavidades torácica e abdominal, bem como do sistema nervoso central, foram coletados e acondicionados em formol tamponado a $10 \%$, clivados, processados rotineiramente, para confecções de lâminas histológicas, que foram coradas por hematoxilina e eosina (HE).

Microscopicamente, as lesões estavam restritas ao cerebelo e caracterizavam-se por alterações neurodegenerativas e necróticas, com desaparecimento segmentar dos neurônios de Purkinje (Fig. 2A).

Vários neurônios de Purkinje apresentavam-se tumefeitos, caracterizados pela presença de pequenos e grandes vacúolos intracitoplasmáticos, verificados também em alguns núcleos dos neurônios afetados. Havia também cromatólise periférica, aumento da eosinofilia citoplasmática, fragmentação e retração nuclear (cariólise e picnose) (Fig. 2B), por vezes com desaparecimento do núcleo e de áreas multifocais com ausência de neurônios de Purkinje. Nessas áreas, também se verificaram espaços em branco, denominados aspecto de cesto vazio, resultantes da perda de neurônios de Purkinje, além de raros esferoides axonais e proliferação dos astrócitos de Bergmann. Em algumas áreas, a camada granular estava hipocelular e havia moderada gliose multifocal na camada molecular. 

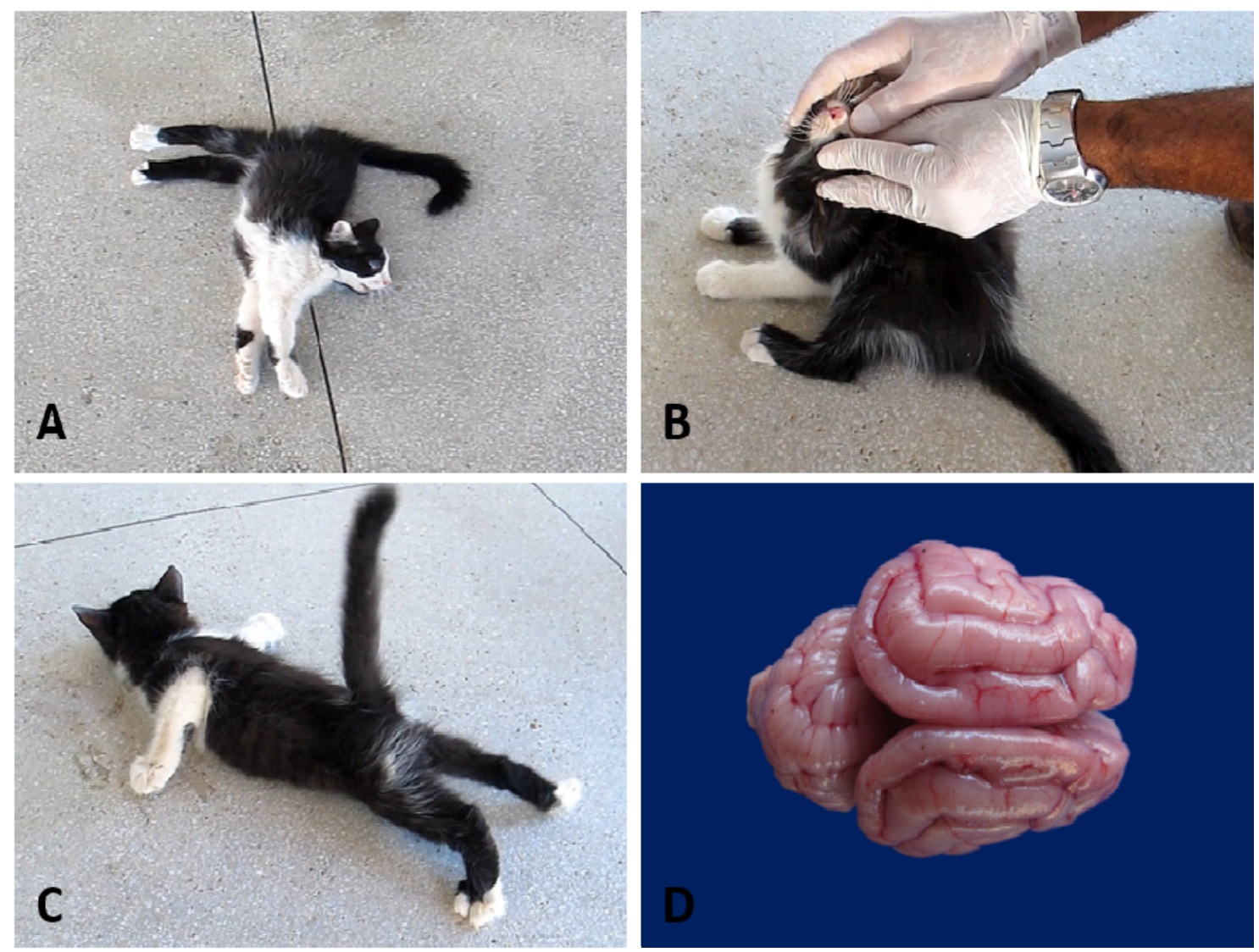

Figura 1. Abiotrofia cerebelar em um gato. A) Felino em decúbito lateral com espasticidade dos membros e opistótono. B) Realização do "head raising test". C) Felino demonstrando espasticidade dos membros e cauda após a realização do "head raising test”. D) Encéfalo sem alterações.
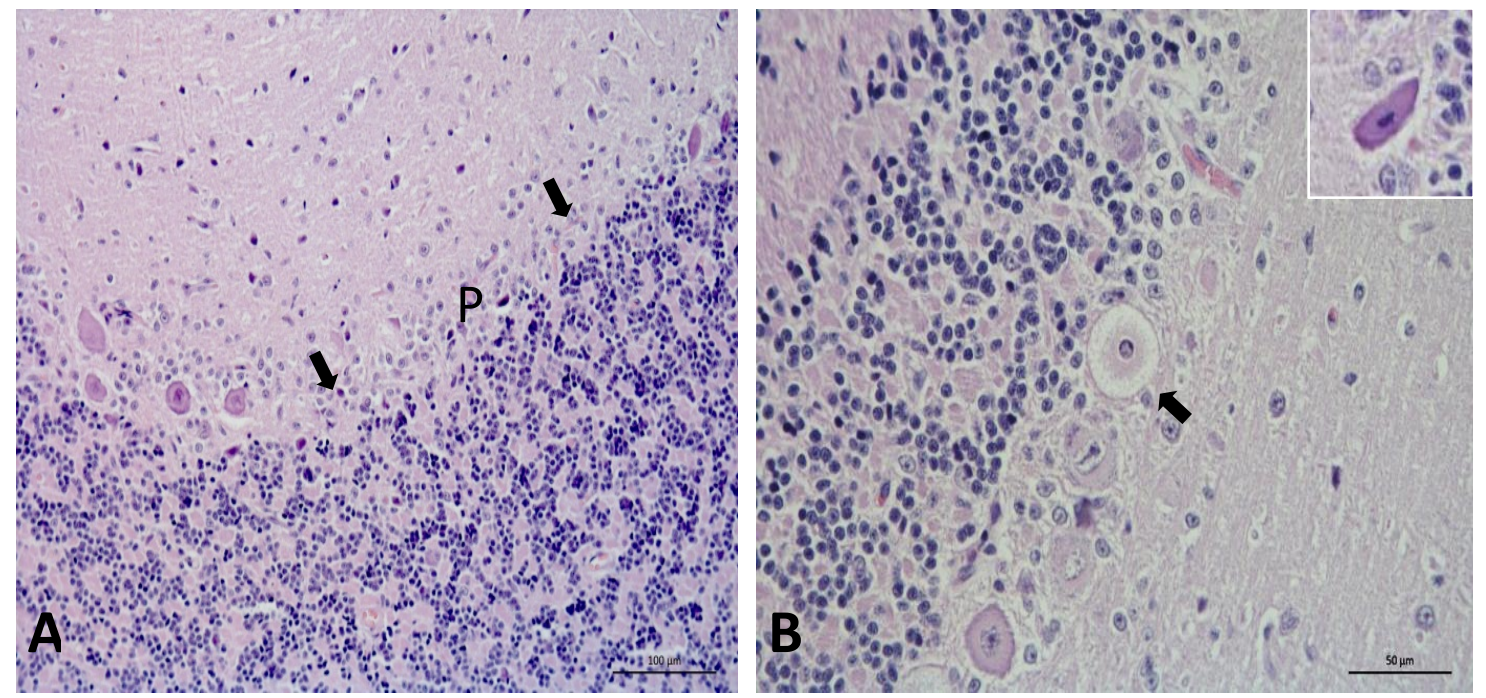

Figura 2. Abiotrofia cerebelar em um gato. A) Ausência de neurônios de Purkinje (P) e astrocitose de Bergmann (setas). HE. B) Observa-se neurônio de Purkinje tumefeito e núcleo picnótico (seta). Em detalhe, observa-se neurônio de Purkinje necrótico. HE 


\section{DISCUSSÃO}

O diagnóstico de abiotrofia cerebelar no presente caso baseou-se nos achados epidemiológicos, nos sinais clínicos e, principalmente, nas lesões histopatológicas observadas no cerebelo que são compatíveis com a doença.

Os animais com essa doença normalmente apresentam sinais de síndrome cerebelar, como observado neste caso e em outras ocorrências de abiotrofia cerebelar descritas em equinos, bovinos, cães (Lahunta 1990; Shamir et al., 1999; Macêdo et al.,2011; Mari et al., 2014) e felinos (Negrin et al., 2006; Biolatti et al., 2010). O diagnóstico da doença é baseado principalmente na presença das lesões neurodegenerativas e necróticas dos neurônios de Purkinje (Mari et al., 2014), mas, além disso, observou-se a diminuição da celularidade da camada granular e astrocitose de Bergmann, como descrito por Negrin et al. (2006) e Biolatti et al. (2010) em felinos. Portanto, as manifestações clínicas, caracterizadas sobretudo por ataxia e tremores de intenção, observados neste animal, são decorrentes das lesões verificadas no cerebelo (Mari et al., 2014).

O exame neurológico utilizando o "head raising test" foi fundamental para direcionar a localização da lesão no cerebelo. Esse teste é frequentemente utilizado em animais de produção (Pienaar et al., 1976) e foi de grande importância na avaliação clínica do paciente, podendo ser incluído com mais frequência na rotina da clínica de pequenos animais nos casos em que os pacientes apresentarem sinais neurológicos sugestivos de disfunção cerebelar.

O início dos sinais clínicos da abiotrofia cerebelar é variável, e a doença apresenta uma evolução clínica progressiva (Ettinger e Feldman, 2004; Biolatti et al., 2010; Pellegrino, 2010). Geralmente cães e gatos manifestam os sinais clínicos entre cinco e oito semanas de idade, como observado neste caso, no qual o animal manifestou sinais com seis semanas de vida. Entretanto, a abiotrofia cerebelar pode se manifestar logo após o nascimento ou de forma tardia (Lahunta e Glass, 2009; Pellegrino, 2010). A abiotrofia cerebelar pós-natal é mais comum e tem sido relatada em várias raças de cães e gatos (Lahunta e Glass, 2009). A forma tardia acontece raramente e tem sido relatada em cães
(Pellegrino, 2010) e gatos (Negrin et al., 2006; Biolatti et al., 2010).

Geralmente não são observadas lesões macroscópicas cerebelares nos animais afetados, porém raramente o cerebelo pode apresentar-se discretamente diminuído de tamanho e achatado (Cantile e Youssef, 2016). A ausência de lesões macroscópicas, na maioria dos animais com abiotrofia cerebelar (Riet-Correa et al., 1998; Negrin et al., 2006; Biolatti et al., 2010), possivelmente está relacionada ao curso da doença, pois quanto mais tarde for descoberta, maior será o grau de degeneração celular, e, por conseguinte, a atrofia cerebelar poderá ser evidenciada.

A origem da abiotrofia cerebelar não está totalmente elucidada, entretanto a hipótese sobre o envolvimento genético vem sendo utilizada por muitos autores, apresentando-se potencialmente como causa para o desencadeamento da doença (Pellegrino, 2010; Oliveira, 2012; Mari et al., 2014), que se relaciona a um defeito genético, com uma herança autossômica recessiva como o principal fator para a manifestação da doença, que pode se expressar logo após o nascimento ou de modo tardio (Pellegrino, 2010; Oliveira, 2012). A enfermidade já vinha acometendo os filhotes de duas gatas irmãs, o que enfatiza a hipótese de herança genética envolvida na etiologia do processo.

A doença deve ser diferenciada da hipoplasia cerebelar, que se manifesta com sintomatologia semelhante e que ocorre com uma maior frequência em felinos no período perinatal, associada à infecção intrauterina pelo Parvovirus felino, que provoca o não desenvolvimento adequado do cerebelo, evidenciado macroscopicamente pela acentuada diminuição do órgão (Riet-Correa et al., 1998; Résibois et al., 2007; Poncelet et al., 2013), não verificado neste caso. Além disso, outras doenças degenerativas devem ser incluídas no diagnóstico diferencial, como as doenças de armazenamento lisossomal, distrofia neuroaxonal e encefalopatia espongiforme felina, principalmente na forma tardia da abiotrofia cerebelar (Biolatti et al., 2010).

O diagnóstico da abiotrofia cerebelar apenas por meio de histórico e sinais clínicos é muito complexo e sugere somente a desordem 
cerebelar, por isso ressalta-se a importância da realização do exame histopatológico do cerebelo para identificação das lesões características, demonstrando-se um método eficiente no diagnóstico.

\section{REFERÊNCIAS}

BIOLATTI, C.; GIANELLA, P.; CAPUCCHIO, M.T. et al. Late onset and rapid progression of cerebellar abiotrophy in a domestic shorthair cat. J. Small Anim. Pract., v.51, p.123-126, 2010.

CANTILE, C.; YOUSSEF, S. Nervous system. In: MAXIE, M.G. Jubb, Kennedy and Palmer's pathology of domestic animals. 6.ed. St. Louis, Missouri: Elsevier, 2016. v.1, p.250-406.

LAHUNTA, A. Abiotrophy in domestic animals: a review. Can. J. Vet. Res., v.54, p.65-76, 1990.

LAHUNTA, A.; GLASS, E. Veterinary neuroanatomy ad clinical neurology. 3.ed. St Louis: Elsevier, 2009. 600p.

ETTINGER, J.S.; FELDMAN, C.E. Tratado de medicina interna veterinária: doenças do cão e do gato. 5.ed. Rio de Janeiro: Guanabara Koogan, 2004.

FOLEY, A.; GRADY, J.; ALMES, K. et al. Cerebellar abiotrophy in a 6-year-old Arabian mare. Equine Vet. Educ., v.23, p.130-134, 2011.

MACÊDO, J.T.S.A.; LUCENA, R.B.; GIARETTA, P.R. et al. Defeitos congênitos em bovinos da Região Central do Rio Grande do Sul. Pesqui. Vet. Bras., v.31, p.297-306, 2011.

MARI, C.; BASSUINO, D.M.; WOUTERS, A.T.B. et al. Abiotrofia cerebelar em um canino American Staffordshire Terrier adulto no Brasil. Acta Sci. Vet., v.42, p.1-5, 2014.
NEGRIN, A.; BERNARDINI, M.; BAUMGARTNER, W. et al. Late onset cerebellar degeneration in a middle-aged cat. $J$. Feline Med. Surg., v.8, p.424-429, 2006.

OLIVEIRA, T.S. Diagnóstico diferencial das enfermidades do sistema nervoso de bovinos no serviço defesa sanitária de Minas Gerais. 2012. 57f. Dissertação (Mestrado em Ciência Animal) Faculdade de Veterinária, Universidade Federal de Minas Gerais, Belo Horizonte, MG.

PELLEGRINO, F.C. Enfermidades degenerativas cerebelosas. Rev. Argent. Neurol. Vet., v.1, p.5058,2010 .

PIENAAR, J.G.; KELLERMAN, T.S.; BASSON, P.A. et al. Maldronksiekte in cattle: A neuronopathy caused by Solanum Kwebense N.E. Br. Onderstepoort. J. Vet. Res., v.43, p.6774, 1976.

PONCELET, L.; HÉRAUDB, C.; SPRINGINSFELDA, M. et al. Identification of feline panleukopenia virus proteins expressed in Purkinje cell nuclei of cats with cerebellar hypoplasia. Vet. J., v.196, p.381-387, 2013.

RÉSIBOIS, A.; COPPENS, A.; PONCELET, L. Naturally occurring Parvovirus-associated feline hypogranular cerebellar hypoplasia-a comparison to experimentally-induced lesions using immunohistology. Vet. Pathol. v.44, p.831-841, 2007.

RIET-CORREA, F.; SCHILD, A.L.; FERNANDES, C.G. Enfermidades do sistema nervoso dos ruminantes no Sul do Rio Grande do Sul. Ciênc. Rural, v.28, p.341-348, 1998.

SHAMIR, M.; PERL, S.; SHARON, L. Late onset of cerebellar abiotrophy in a Siamese cat. J. Small Anim. Pract., v.40, p.343-345, 1999. 